\title{
An Analysis of a Wind Turbine-Generator System in the Presence of Stochasticity and Fokker-Planck Equations
}

\author{
Ravish Himmatlal Hirpara, S.V. National Institute of Technology (SVNIT), Surat, India \\ https://orcid.org/0000-0003-1222-3562
}

Shambhu Nath Sharma, S.V. National Institute of Technology (SVNIT), Surat, India

\begin{abstract}
In power systems dynamics and control literature, theoretical and practical aspects of the wind turbine-generator system have received considerable attentions. The evolution equation of the induction machine encompasses a system of three first-order differential equations coupled with two algebraic equations. After accounting for stochasticity in the wind speed, the wind turbine-generator system becomes a stochastic system. That is described by the standard and formal Itô stochastic differential equation. Note that the Itô process is a strong Markov process. The Itô stochasticity of the wind speed is attributed to the Markov modeling of atmospheric turbulence. The article utilizes the Fokker-Planck method, a mathematical stochastic method, to analyse the noise-influenced wind turbine-generator system by doing the following: (i) the authors develop the Fokker-Planck model for the stochastic power system problem considered here; (ii) the Fokker-Planck operator coupled with the Kolmogorov backward operator are exploited to accomplish the noise analysis from the estimation-theoretic viewpoint.
\end{abstract}

\section{KEYWORDS}

Differential Equations (SDEs), Fokker-Planck Technique, Small Scale Turbulence, Stochastic, Wind TurbineGenerator System

\section{INTRODUCTION}

The wind energy has become one of the popular renewable sources for the generation of the electrical power. Three different configurations are popular in the wind power conversion technology, the fixed speed wind turbine-generator system, the limited variable speed wind turbine-generator system and variable speed wind turbine-generator system. The main components of the fixed speed wind turbine-generator system are wind turbine, gearboxes, squirrel cage induction generator, capacitor. That are connected to the grid through the transformer. This configuration allows the machine to run at the constant speed as well as provides stable frequency. Secondly, the limited variable speed wind turbine-generator system utilizes the wound rotor induction generator in place of the squirrel cage. This structure utilizes a power electronic converter. Thirdly, the variable speed wind turbine-generator system accounts for the doubly-fed induction generator with a power electronic converter ( $\mathrm{Li}$ and $\mathrm{Chen}$, 2008). Here, some important dynamical equations for the wind turbine-generator system are described. Fu and Xing (2009) have developed a four-dimensional Squirrel Cage Induction Generator (SCIG) 
model. Subsequently, a reduced-order model was developed using singular perturbation method. The singular perturbation method hinges on the electrical engineering assumption that stator transients are faster than rotor transients. Thus, the evolutions of stator transients are not accounted for and the evolutions of rotor transients are accounted for. Taha Hussien et al. (2017) have developed modeling of three phase induction motor and explain stator turns fault analysis based on artificial intelligence. Salima et al. (2018) have developed a Global stability of linearizing control of induction motor for PV water pumping application. To study the control system design using Matlab see (Moysis et al. 2017). Gabhi et al. (2018) have developed discrete sliding mode control scheme for nonlinear systems with bounded uncertainties and Alain et al. (2017) have developed improved robust adaptive control strategy for the finite time synchronization of uncertain nonlinear systems. Feijóo et al. (2000) have considered a third-order deterministic equation that explains modelling of the rotor transient voltages behind the transient impedance. Balanathan et al. (2002) have developed a dynamic load model. That has ability to analyse the transient and steady state stabilities of the induction motor. To study the influence of the wind power dynamics on the large electrical power systems dynamics and control, Slootweg et al. (2003) argue the addition of wind turbine-generating systems' models in software packages, especially for numerical experimentations. In this connection, especially for numerical experimentations of wind turbine models, two articles, i.e. Sorensen et al. (2002) and Nichita et al. (2002), would be also useful. Dusonchet et al. (2007) have investigated the influence of wind turbine mechanical characteristics. The transient voltage stability of the fixed speed wind turbine-generator systems were studied as well. In their investigation, the fifth-and the third-order models were the subject of investigations. Generally, the third-order model is a special case of the fifth-order model. The third-order does not account for stator transients into considerations, see Popović et al. (1998) and Ledesma et al. (2003). Furthermore, Martins et al. (2007) have validated the fifth-and thirdorder models with measured data, which are rarely reported in literature. The preceding introductory descriptions of this paper suggest that the problem of analysing the wind turbine-generator system from the dynamical systems' viewpoint received considerable attention in literature. Under the wind turbulence influence, it is reasonable to account for the stochastic character of the wind speed for the wind turbine-generator system in lieu of the deterministic formalism. A good discussion about the wind turbine-generator system under stochastic influence can be found in brief research writing, but a compelling paper in stochastic sense (Wang and Crow 2012). The stochastically perturbed dynamical system involves investigations into vector Stochastic Differential Equations (SDEs) in lieu of Ordinary Differential Equations (ODEs). This seems to be one of the reasons that the notion of stochastic processes is relatively very less introduced into 'power systems dynamics' literature yet.

This paper is aimed to account for the stochastic evolution of the wind speed in lieu of the deterministic for the wind turbine-generator system. This paper is inspired from the fact that the systems and control methods under stochastic considerations contribute to the greater accuracy and refinements (Mumford 2000) in available results, see Sinai (1981). Here, a succinct discussion that the Itô stochasticity is imperative for the wind turbine-generator system. The turbulent atmospheric flows influences the wind speed operation, the response time of wind turbines is typically in the range of seconds. Thus, they are subjected to small scale turbulence. The Fokker-Planck analysis for small scale turbulence agree with experimental corroborations (Renner et al. 2001). From the theory of stochastic processes, the Fokker-Planck equation is a consequence of the Itô stochastic differential equation. Thus, the qualitative characteristics of atmospheric turbulence are embedded in the Itô stochastic correction term of the wind speed evolution equation. The major ingredient of this paper, which is utilized to develop the theory of this paper, is the Fokker-Planck equation. The Fokker-Planck equation is an influential result in the theory of stochastic processes and stochastic differential equations. The equation is the evolution of conditional probability density for a given initial state for a Markovian state vector. In Wang and Crow (2012), the 'reduced-order' Fokker-Planck equation for the two-dimensional state comprising the slip and wind speed, was the subject of investigations. In their analysis, the numerical experimentation for the reduced Fokker-Planck equation was accomplished 
under a variety of conditions. In Wang and Crow (2012), numerical simulations for the stochastic wind turbine system were achieved in the conditional probability sense. In contrast to Wang and Crow (2012), the estimation of the wind turbine-generator system for the first time using the Itô stochastic interpretation and Fokker-Planck equation are achieved. It is important to note that the stochastic evolution of the conditional expectation of the scalar function is the cornerstone formalism of estimation theory and stochastic processes. Notably, the Fokker-Planck equation for the wind turbinegenerator system involving the four-dimensional state vector are revisited. The four dimensional state vector has the following components: the wind speed, slip, two voltages behind transient impedance in $d-q$ reference frame. Furthermore, the Fokker-Planck model of the stochastic problem of concerned here is utilized to accomplish the noise analysis of the stochastic problem from the estimation-theoretic viewpoint. This paper is the first paper in the sense that this paper introduces the notion of the Fokker-Planck equation into power systems dynamics in a greater detail.

\section{THE WIND TURBINE GENERATOR STOCHASTIC DYNAMICS AND ITS FOKKER-PLANCK EQUATION}

The induction machine transient dynamics is described by a system of three first-order differential equations coupled with two algebraic equations. Furthermore, the wind turbine-generator dynamics accounts for the wind speed as a state variable. More precisely, the wind turbine-generator system becomes a multi-dimensional dynamical system, where the dimension of the state vector becomes four. In the stochastic framework, the wind turbine-generator system becomes a multi-dimensional stochastic differential system. The stochasticity is attributed to considering the wind speed as a random variable. The dimension of the state vector, which describes the fixed speed wind turbine-generator system dynamics, is four. For the sake of clarity, a schematic diagram of a wind turbine generator system is illustrated in a simple, but a revealing Figure 1.

A greater detail about the deterministic dynamics can be found in Kundur (1994) and Wu et al. (2011). The procedure is lengthy as well as this paper is not aimed to revisit the deterministic dynamics of the wind turbine-generator system. State directly the wind turbine-generator deterministic dynamics. Making the use of general theory of Induction Machines in $d-q$ reference frame, electromagnetic induction principle, we arrive at the following system of dynamical equations of the wind turbinegenerator system:

Figure 1. A wind turbine-generator system

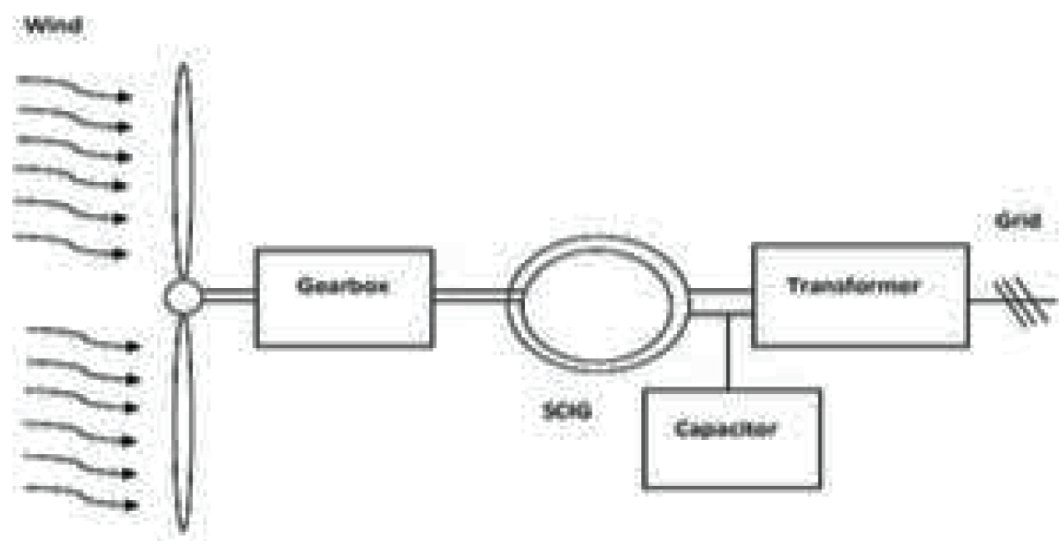




$$
\begin{aligned}
& \dot{v}_{t}=\beta\left(\frac{\sigma^{2}}{v_{t}}-v_{t}\right) \\
& \dot{s}=\frac{1}{J_{t o t} \omega_{s}^{2}}\left(P_{e}-\frac{P_{m}}{(1-s)}\right) \\
& \dot{e}_{d}^{\prime}=s \omega_{s} e_{q}^{\prime}-\frac{1}{T_{0}^{\prime}}\left(e_{d}^{\prime}+\left(X_{s}-X_{s}^{\prime}\right) i_{q s}\right) \\
& \dot{e}_{q}^{\prime}=-s \omega_{s} e_{d}^{\prime}-\frac{1}{T_{0}^{\prime}}\left(e_{q}^{\prime}-\left(X_{s}-X_{s}^{\prime}\right) i_{d s}\right)
\end{aligned}
$$

where:

$$
\begin{aligned}
& e_{d}^{\prime}=-R_{s} i_{d s}+X_{s}^{\prime} i_{q s}+v_{d s} \\
& e_{q}^{\prime}=-R_{s} i_{q s}-X_{s}^{\prime} i_{d s}+v_{q s} \\
& P_{e}=-e_{d}^{\prime} i_{d s}-e_{q q s}^{\prime} i_{q_{s}} \\
& Q_{e}=e_{q d s}^{\prime} i_{d s}-e_{d}^{\prime} i_{q s}
\end{aligned}
$$

$$
P_{m}=\frac{1}{2} \rho \pi R^{2} C_{p}(\lambda, \theta) v_{t}^{3}
$$

$$
\begin{aligned}
& X_{s}=\omega_{s}\left(L_{s}+L_{m}\right), X_{s}^{\prime}=\omega_{s}\left(L_{s}+L_{m}-\frac{L_{m}^{2}}{L_{r}+L_{m}}\right) \\
& e_{d}^{\prime}==-\omega_{s} \frac{L_{m}}{L_{m}+L_{r}} \phi_{q r}, e_{q}^{\prime}==\omega_{s} \frac{L_{m}}{L_{m}+L_{r}} \phi_{d r}, T_{0}^{\prime}=\frac{L_{m}+L_{r}}{R_{r}}
\end{aligned}
$$


Note that the dot sign associated with the above set of equations denotes the time derivative. The wind turbine-generator system is described by a four-dimensional state vector and involves a larger number of system parameters. Here, we explain succinctly the notational machinery of system parameters of the dynamical system considered here. The terms $v_{t}, s, e_{d}^{\prime}, e_{q}^{\prime}$ denote the wind speed, slip and $d$-axis voltage behind transient impedance and $q$-axis voltage behind transient impedance respectively. The induction machine circuit parameters $L_{s}, L_{r}$ denote the stator and rotor inductances respectively. Similarly, the circuit parameters $R_{s}, R_{r}, L_{m}, \phi_{d r}, \phi_{q r}$ are stator resistance, rotor resistance, mutual inductance, $d$-axis rotor flux linkage and, $q$-axis rotor flux linkage the induction machine respectively.

Furthermore, the system parameters vector $\left(s, \omega_{s}, \rho, C_{p}(\lambda, \theta), R, J_{t o t}\right)^{T}$ denotes slip, synchronous speed, air density, power coefficient, turbine radius, and system moment of inertia respectively:

$$
C_{p}(\lambda, \theta)=c_{1}\left(\frac{c_{2}}{\mu}-c_{3} \theta-c_{4} \theta^{c_{5}}-c_{6}\right) e^{-\frac{c_{7}}{\mu}}, \mu=\frac{1}{\frac{1}{\lambda+c_{8} \theta}-\frac{c_{9}}{\theta^{3}+1}}
$$

The notation $C_{p}(\lambda, \theta)$ denotes performance coefficient or power coefficient $\lambda$ is a tip speed ratio, the ratio between the blade tip speed and wind speed, $\theta$ is a pitch angle in degree. See Slootweg et al. (2003) for a greater detail. Note that Equations (1a)-(1i) constitute Equation (1).

Here, we explain how the atmospheric turbulence introduces fluctuations into the wind speed. The relative motion of fluid particles under the influence of turbulence can be described as Stochastic Differential Equations (SDEs), see the SDE of Pedrizzetti and Novikov (1994). The atmospheric turbulence introduces stochasticity into the wind speed, generator rotor as well as the wind energy power output (Milan et al. 2013, Wächter et al. 2012). See Mücke et al. (2015), Karlsen (2006, p.8), Friedrich and Peinke (1997) and Wang and Crow (2012, p.2) as well. That suggest stochastic models are imperative for the wind turbine-generator system. Friedrich et al. (2011) discussed about the choice of stochastic processes to model the qualitative characteristics of complex systems arising from diverse fields. The Markov process has proven useful to model small scale turbulence (Renner et al. 2001, Pedrizzetti and Novikov 1994). Analytical solution as well as numerical simulations to the Fokker-Planck equation for small scale turbulence agree with experimental corroborations (Renner et al. 2001). A N Kolmogorov constructed the third-order statistics for small scale turbulence that hinges on the Navier-Stokes' equation (Kolmogorov 1941), see Kolmogorov (1962) as well. Wind turbines operate under the influence of the turbulent atmospheric flows and the response time of wind turbines is typically in the range of seconds, thus, they are subjected to small scale turbulence. Small-scale homogeneous isotropic turbulence has an important impact on the wind energy conversion process (Wächter et al. 2012). Secondly, numerical as well as actual experimentations are the indicative of Markov processes to model small scale turbulence. For these two reasons, a non-linear Markov process to model stochasticity in the wind speed is adopted, i.e.:

$$
d v_{t}=\beta\left(\frac{\sigma^{2}}{v_{t}}-v_{t}\right) d t+\gamma \sigma \sqrt{2 \beta} d B_{t}
$$

where the terms $v_{t}$ and $B_{t}$ denote the wind speed and the Brownian motion. Note that the wind speed $v_{t}$ is a non-linear Markov process for two reasons. (i) the above stochastic differential equation describes a non-linear system, which is driven by a Brownian motion and initial datum. A revealing and an alternative structure of the square of the stochastic wind speed $v_{t}$ satisfies the following SDE: 


$$
\begin{aligned}
& d v_{t}^{2}=2 v_{t} d v_{t}+\left(d v_{t}\right)^{2}=2 \beta\left(\sigma^{2}-v_{t}^{2}\right) d t+2 \beta \gamma^{2} \sigma^{2} d t+2 \gamma \sigma \sqrt{2 \beta} v_{t} d B_{t} \\
& =\left(2 \beta\left(\sigma^{2}-v_{t}^{2}\right)+4 \beta \gamma^{2} \sigma^{2}\right) d t+2 \gamma \sigma \sqrt{2 \beta} v_{t} d B_{t}
\end{aligned}
$$

The equation is a consequence of Itô stochastic differential rules, i.e.:

$$
\begin{aligned}
& v_{t} d v_{t}=\beta\left(\sigma^{2}-v_{t}^{2}\right) d t+\gamma \sigma \sqrt{2 \beta} v_{t} d B_{t},\left(v_{t} d v_{t}\right)^{2}=2 \beta \gamma^{2} \sigma^{2} v_{t}^{2} d t \\
& \left(d v_{t}\right)^{2}=2 \beta \gamma^{2} \sigma^{2} d t
\end{aligned}
$$

Choose $y_{t}=v_{t}^{2}$, we get:

$$
d y_{t}=\left(2 \beta\left(\sigma^{2}-y_{t}\right)+2 \beta \gamma^{2} \sigma^{2}\right) d t+2 \gamma \sigma \sqrt{2 \beta} \sqrt{y_{t}} d B_{t}
$$

The equation is a non-linear Itô stochastic differential equation, where non-linearity is attributed to the coupling term $\sqrt{y_{t}} d B_{t}, y_{t}=v_{t}^{2}$. The output is a state variable wind speed. (ii) The above stochastic differential equation is an Itô stochastic differential equation and the Itô process is always Markov process. For this case, the wind speed is an Itô process and becomes the non-linear Markov process. Since formulation hinges on the Markov process and the Fokker-Planck equation, results hold for stochasticity of the wind turbine-generator system attributed to the small scale turbulence. After considering the stochastic correction term in the wind speed (Wang and Crow 2012), Equations (1a)-(1d) can be further recast in a formal non-linear Markovian stochastic setting as:

$$
\begin{aligned}
& d v_{t}=\beta\left(\frac{\sigma^{2}}{v}-v\right) d t+\gamma \sigma \sqrt{2 \beta} d B_{t} \\
& d s=\frac{1}{J_{t o t} \omega_{s}^{2}}\left(P_{e}-\frac{P_{m}}{(1-s)}\right) d t \\
& d e_{d}^{\prime}=\left(s \omega_{s} e_{q}^{\prime}-\frac{1}{T_{0}^{\prime}}\left(e_{d}^{\prime}+\left(X_{s}-X_{s}^{\prime}\right) i_{q s}\right)\right) d t \\
& d e_{q}^{\prime}=\left(-s \omega_{s} e_{d}^{\prime}-\frac{1}{T_{0}^{\prime}}\left(e_{q}^{\prime}-\left(X_{s}-X_{s}^{\prime}\right) i_{d s}\right)\right) d t
\end{aligned}
$$

Note that the term $v_{t}$ of Equation (2a) is a Rayleigh process in the mathematical framework. The term $v_{t}$ is the wind speed in the context of engineering systems as well. A connection between the Rayleigh process and wind speed is nicely explained in Wang and Crow (2012). Since this paper 
is chiefly intended to analyse the stochastic system concern here in the Fokker-Planck setting, further discussions on Equation (2) are omitted of the paper. Note that a theoretical interpretation of the white noise process is the Itô setting. In the Itô setting, the term $d B_{t}=w_{t} d t$ is the subject of investigations (Kuo 2005, Kunita 2010).

The Itô SDE is a convenient form for stochastic control problems (Jazwinski 1970, Kushner 1967). The stochastic control perspective of dynamic systems encompasses the following: (i) estimations (ii) stochastic stability (iii) control algorithms.

The term $w_{t}$ is a white noise process and the term $B_{t}$ denotes the Brownian process. The Fokker-Planck equation is the cornerstone formalism to analyse the stochasticity of the wind-turbine generator system of this paper. The Fokker-Planck equation is a parabolic linear homogeneous equation of order two in partial differentiation for the transition probability density of the Ito stochastic differential equation. This reveals a connection between stochastic differential equations and partial differential equations. The Fokker-Planck operator, alternatively known as the Kolmogorov-FokkerPlanck operator, is a linear operator. The above set of scalar dynamical equations can be recast as the vector Itô stochastic differential equation. Thus, the equations become (Jazwinski 1970):

$$
d x_{t}=f\left(x_{t}, t\right) d t+G\left(x_{t}, t\right) d B_{t}
$$

For simplified analysis, we adopt more familiar notations of SDEs, i.e.:

$$
\begin{aligned}
& x_{t}=\left(x_{i}(t)\right)_{1 \leq i \leq 4}=\left(v_{t}, s, e_{d}^{\prime}, e_{q}^{\prime}\right)^{T}=\left(x_{1}, x_{2}, x_{3}, x_{4}\right)^{T} \\
& \beta\left(\frac{\sigma^{2}}{x_{1}}-x_{1}\right) \\
& f\left(x_{t}, t\right)=\left(\begin{array}{c}
\rho \pi R^{2} \\
\frac{P_{e}}{J_{t o t} \omega_{s}^{2}}-\frac{1}{2 J_{t o t} \omega_{s}^{2}\left(1-x_{2}\right)} C_{p}(\lambda, \theta) x_{1}^{3} \\
x_{2} \omega_{s} x_{4}-\frac{1}{T_{0}^{\prime}}\left(x_{3}+\left(X_{s}-X_{s}^{\prime}\right) i_{q s}\right) \\
-x_{2} \omega_{s} x_{3}-\frac{1}{T_{0}^{\prime}}\left(x_{4}-\left(X_{s}-X_{s}^{\prime}\right) i_{d s}\right)
\end{array}\right), G\left(x_{t}, t\right)=G(t)=\left(\begin{array}{c}
\gamma \sigma \sqrt{2 \beta} \\
0 \\
0 \\
0
\end{array}\right)
\end{aligned}
$$

The terms $f\left(x_{t}, t\right)$ and $G\left(x_{t}, t\right)$ are the system non-linearity and the dispersion matrix of the stochastic differential equation respectively. Note that the notation $\left(x_{1}, x_{2}, x_{3}, x_{4}\right)^{T}$ will be utilized for the state vector of the stochastic system considered here throughout the paper in lieu of the notation $\left(v_{t}, s, e_{d}^{\prime}, e_{q}^{\prime}\right)^{T}$. More importantly, Equation (3) describes the wind turbine-generator Itô SDE, the cornerstone formalism of this paper. Here, the dimensionality and non-linearity of the wind turbinegenerator stochastic system are explained. For linear systems, the state and system parameters can be decoupled that lead to the relation $f\left(x_{t}, t\right)=A_{t} x_{t}$. More precisely, for linear cases, the matrixvector format arises, on the other hand, the vector interpretation holds for non-linearity. Since the stochastic system of the paper is non-linear, the state and system parameters cannot be decoupled, the system non-linearity will have vector format in lieu of the matrix, i.e. $f\left(x_{t}, t\right)=\left(f_{i}\left(x_{t}, t\right)\right)_{1 \leq i \leq 4}$. Secondly, the random input associated with the stochastic system is scalar, the process noise coefficient is a vector, i.e. $G\left(x_{t}, t\right)=\left(G_{i \varphi}(t)\right)_{1 \leq i \leq n, 1 \leq \varphi \leq 1}=\left(G_{i}(t)\right)_{1 \leq i \leq n}$, in place of the matrix format. Equation (3) can be treated as an advanced and a refined system of dynamical equations in contrast to the 
deterministic setting. Equation (3) describes a non-linear Itô SDE. Other 'setting' of stochastic differential systems is the Stratonovich. Interestingly, the structure of Equation (2) remains invariant under the both settings, since the stochasticity associated is the state-independent, see the last term of Equation (2a) of the paper. The non-linearity in the system dynamics considered here is attributed to a non-linear evolution stated in Equation (2b). The stochasticity is attributed to the random forcing term associated with the wind speed evolution equation, see Equation (2a). One can arrive at Equation (2) using the electromagnetic induction principle of electrical machinery, the concepts of stochastic processes. Some simplifications are coupled with assumptions that stator transients are ignored and the rotor circuit is short circuited. The system of stochastic differential equations utilizes the notion of $d-q$ reference frame, where the both axes are orthogonal to each other. One can arrive at the wind turbine-generator system dynamics using the concept of Lagrangian mechanics as well. The procedure involves writing down the Lagrangian of the dynamical system and subsequently, we write down the Euler-Lagrange equation. Here, we will not delve into Lagrangian mechanics in the context of the wind turbine-generator system. A brief discussion about the Lagrangian formulation in the context of electrical machines can be found in Pintea et al. (2011), Greets et al. (2005).

It is important to note that after considering stator transient dynamics, we are led to a sixdimensional random state vector. That introduces the dimensional formidable complexity. The fourdimensional system of dynamical equations has proven useful in literature for the sake of simplified analysis, e.g. Wang and Crow (2012). Thus, Equation (3) would be the subject of investigations in this paper (see Figure 2).

The structure of the wind turbine-generator system stated in Equation (3) remains invariant in different configurations of the wind turbine-generator system. Importantly, the additional correction term attributed to the rotor voltage is accounted for in variable speed machines, see Feijóo et al. (2000). On the other hand, the rotor voltage vanishes for fixed speed machines. Secondly, the dimension of the state vector will be same in fixed and variable speed machines.

The Fokker-Planck equation, a parabolic linear homogeneous equation, is a systems and control method in the theory of stochastic control. For a good access about the Fokker-Planck equation, see Bierbaum et al. (2002), Risken (1984), Sharma (2008), and Chow et al. (2007). After applying 'the Kolmogorov-Fokker-Planck equation for Markov processes (Karatzas and Shreve 1988) to the randomly perturbed system of the paper, we get:

$$
d p=\left(-\sum_{i} \frac{\partial f_{i}(x, t) p}{\partial x_{i}}+\frac{1}{2} \sum_{i, j} \frac{\partial^{2}\left(G G^{T}\right)_{i j}(x, t) p}{\partial x_{i} \partial x_{j}}\right) d t
$$

Figure 2. A wind turbine generator system SDE diagram

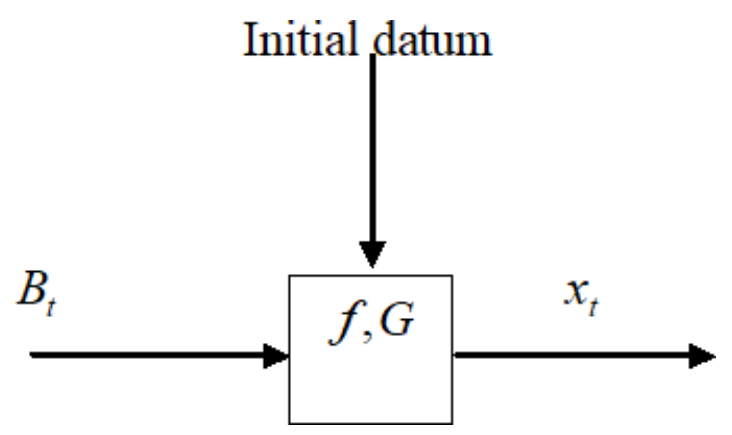




$$
\begin{aligned}
& =\left(-\frac{\partial \beta\left(\frac{\sigma^{2}}{x_{1}}-x_{1}\right) p}{\partial x_{1}}-\frac{\partial \frac{1}{J_{t o t} \omega_{s}^{2}}\left(P_{e}-\frac{P_{m}}{1-x_{2}}\right) p}{\partial x_{2}}\right. \\
& -\frac{\partial}{\partial x_{3}}\left(x_{2} \omega_{s} x_{4}-\frac{1}{T_{0}^{\prime}}\left(x_{3}+\left(X_{s}-X_{s}^{\prime}\right) i_{q s}\right)\right) p \\
& -\frac{\partial}{\partial x_{4}}\left(-x_{2} \omega_{s} x_{3}-\frac{1}{T_{0}^{\prime}}\left(x_{4}-\left(X_{s}-X_{s}^{\prime}\right) i_{d s}\right)\right) p \\
& \left.+\beta \gamma^{2} \sigma^{2} \frac{\partial^{2} p}{\partial x_{1}^{2}}\right) d t
\end{aligned}
$$

where the term $p$ denotes the conditional probability density $p=p\left(x, t \mid x_{t_{0}}, t_{0}\right)$. The KolmogorovFokker-Planck operator $L($.$) , which is a consequence of Equation (4), for the stochastic wind turbine-$ generator system assumes the following structure:

$$
\begin{aligned}
& L(.)=-\sum_{i} \frac{\partial f_{i}(x, t)(.)}{\partial x_{i}}+\frac{1}{2} \sum_{i, j} \frac{\partial^{2}\left(G G^{T}\right)_{i j}(x, t)(.)}{\partial x_{i} \partial x_{j}} \\
& =-\frac{\partial \beta\left(\frac{\sigma^{2}}{x_{1}}-x_{1}\right)(.)}{\partial x_{1}}-\frac{\partial \frac{1}{J_{t o t} \omega_{s}^{2}}\left(P_{e}-\frac{P_{m}}{1-x_{2}}\right)(.)}{\partial x_{2}} \\
& -\frac{\partial}{\partial x_{3}}\left(x_{2} \omega_{s} x_{4}-\frac{1}{T_{0}^{\prime}}\left(x_{3}+\left(X_{s}-X_{s}^{\prime}\right) i_{q s}\right)\right)(.) \\
& -\frac{\partial}{\partial x_{4}}\left(-x_{2} \omega_{s} x_{3}-\frac{1}{T_{0}^{\prime}}\left(x_{4}-\left(X_{s}-X_{s}^{\prime}\right) i_{d s}\right)\right)(.) \\
& +\beta \gamma^{2} \sigma^{2} \frac{\partial^{2}(.)}{\partial x_{1}^{2}}
\end{aligned}
$$

The Kolmogorov backward operator $L^{\prime}($.$) for the Itô SDE is the adjoint of the forward operator$ $L\left(\right.$.). The Kolmogorov backward operator $L^{\prime}($.$) becomes:$

$$
\begin{aligned}
& L^{\prime}(.)=\sum_{i} f_{i} \frac{\partial(.)}{\partial x_{i}}+\frac{1}{2} \sum_{i, j}\left(G G^{T}\right)_{i j}(x, t) \frac{\partial^{2}(.)}{\partial x_{i} \partial x_{j}} \\
& =\beta\left(\frac{\sigma^{2}}{x_{1}}-x_{1}\right) \frac{\partial(.)}{\partial x_{1}}+\frac{1}{J_{t o t} \omega_{s}^{2}}\left(P_{e}-\frac{P_{m}}{1-x_{2}}\right) \frac{\partial(.)}{\partial x_{2}}
\end{aligned}
$$




$$
\begin{aligned}
& +\left(x_{2} \omega_{s} x_{4}-\frac{1}{T_{0}^{\prime}}\left(x_{3}+\left(X_{s}-X_{s}^{\prime}\right) i_{q s}\right)\right) \frac{\partial}{\partial x_{3}}(.) \\
& +\left(-x_{2} \omega_{s} x_{3}-\frac{1}{T_{0}^{\prime}}\left(x_{4}-\left(X_{s}-X_{s}^{\prime}\right) i_{d s}\right)\right) \frac{\partial}{\partial x_{4}}(.) \\
& +\beta \gamma^{2} \sigma^{2} \frac{\partial^{2}(.)}{\partial x_{1}^{2}}
\end{aligned}
$$

Making the use of the Kolmogorov-Fokker-Planck operator of Equation (5a), Kolmogorov backward operator, Equation (5b), and the definition of the evolution of the conditional expectation of the scalar function of the state $x_{t}$, we arrive at exact evolutions of conditional mean and variance. Suppose the vector system non-linearity $f\left(x_{t}, t\right)$, and the diffusion coefficient matrix $G G^{T}\left(x_{t}, t\right)$ are bounded continuous. Consider the vector system non-linearity has bounded continuous triple derivatives. The diffusion coefficient matrix $G G^{T}\left(x_{t}, t\right)$ has bounded continuous double derivatives.

The following conditional mean and variance evolution equations will be useful for the noise analysis of the non-linear wind turbine-generator system of this paper:

$$
\begin{aligned}
& d\left\langle x_{i}(t)\right\rangle=\left(f_{i}\left(\left\langle x_{t}\right\rangle, t\right)+\frac{1}{2} \sum_{p, q} P_{p q} \frac{\partial^{2} f_{i}\left(\left\langle x_{t}\right\rangle, t\right)}{\partial\left\langle x_{p}\right\rangle \partial\left\langle x_{q}\right\rangle}\right) d t \\
& d P_{i j}=\left(\sum_{p} P_{i p} \frac{\partial f_{j}\left(\left\langle x_{t}\right\rangle, t\right)}{\partial\left\langle x_{p}\right\rangle_{p}}+\sum_{p} P_{j p} \frac{\partial f_{i}\left(\left\langle x_{t}\right\rangle, t\right)}{\partial\left\langle x_{p}\right\rangle}\right. \\
& +\frac{1}{2} \sum_{p, q, r} P_{i p} P_{q r} \frac{\partial^{3} f_{j}^{f}\left(\left\langle x_{t}\right\rangle, t\right)}{\partial\left\langle x_{p}\right\rangle \partial\left\langle x_{q}\right\rangle \partial\left\langle x_{r}\right\rangle}+\frac{1}{2} \sum_{p, q, r} P_{j p} P_{q r} \frac{\partial^{3} f_{i}\left(\left\langle x_{t}\right\rangle, t\right)}{\partial\left\langle x_{p}\right\rangle \partial\left\langle x_{q}\right\rangle \partial\left\langle x_{r}\right\rangle} \\
& \left.+\left(G G^{T}\right)_{i j}\left(\left\langle x_{t}\right\rangle, t\right)+\frac{1}{2} \sum_{p, q} P_{p q} \frac{\partial^{2}\left(G G^{T}\right)_{i j}\left(\left\langle x_{t}\right\rangle, t\right)}{\partial\left\langle x_{p}\right\rangle \partial\left\langle x_{q}\right\rangle}\right) d t
\end{aligned}
$$

where the state $x_{t}=\left(x_{i}(t)\right)$ and the vector system non-linearity $f\left(x_{t}, t\right)=\left(f_{i}\left(x_{t}, t\right)\right)$ and $\left\langle x_{i}\right\rangle=E\left(x_{i}(t) \mid x_{t_{0}}, t_{0}\right), P_{i j}=\left\langle\left(x_{i}-\left\langle x_{i}\right\rangle\right)\left(x_{j}-\left\langle x_{j}\right\rangle\right)\right\rangle$. The notation ' \langle\rangle ' denotes the action of the conditional expectation operator $E$ on random variables. For deterministic initial states, the operator $E$ becomes the expectation in lieu of the conditional expectation.

A proof of the exact estimation equations and a special case of the exact estimation equations, Equations (6a)-(6b), is explained in the Appendix. Equations (6a)-(6b) are available in recently published papers as well, see Sharma (2008) and Equations (1)-(2) of Patel and Sharma (2014). The main intend to analyse the multidimensional stochastic system considered here in the Fokker-Planck setting in lieu of filtering framework. The Fokker-Planck setting is useful for the noise analysis of stochastic systems with 'valueless' observations. On the other hand, the filtering framework is useful for the noise analysis of stochastic systems with 'useful' observations. 
Note that Equation (6) comprises Equations (6a)-(6b). The system of equations, Equations (6a)-(6b), has ability to account for the contributions of linear terms, square non-linearity as well as third-order non-linearity completely. That preserves some of the qualitative characteristics of further higher-order non-linearities.

After combining Equations (3) and (6), we get the following set of coupled conditional moment evolution equations. The conditional mean evolution $d\left\langle x_{t}\right\rangle$ and the variance evolutions $d P_{t}$, for the stochastic problem of concern here, are:

$d\left\langle x_{t}\right\rangle=A\left(\left\langle x_{t}\right\rangle, P_{t}\right) d t$

$d P_{t}=B\left(\left\langle x_{t}\right\rangle, P_{t}\right) d t$

where:

$$
\begin{aligned}
& A\left(\left\langle x_{t}\right\rangle, P_{t}\right)=\left(A_{i}\left(\left\langle x_{t}\right\rangle, P_{t}\right)\right)_{1 \leq i \leq 4}, B\left(\left\langle x_{t}\right\rangle, P_{t}\right)=\left(B_{i j}\left(\left\langle x_{t}\right\rangle, P_{t}\right)\right)_{\substack{1 \leq i \leq 4 \\
1 \leq j \leq 4 \\
1}} \\
& A_{1}\left(\left\langle x_{t}\right\rangle, P_{t}\right)=\beta\left(\frac{\sigma^{2}}{\left\langle x_{1}\right\rangle}-\left\langle x_{1}\right\rangle\right)+P_{11} \beta \gamma^{2} \sigma^{2} \frac{1}{\left\langle x_{1}\right\rangle^{3}} \\
& A_{2}\left(\left\langle x_{t}\right\rangle, P_{t}\right)=-\frac{1}{2} \frac{\rho \pi R^{2}}{J_{t o t} \omega_{s}^{2}\left(1-\left\langle x_{2}\right\rangle\right)} C_{p}(\lambda, \theta)\left\langle x_{1}\right\rangle^{3}+\frac{\left\langle P_{e}\right\rangle}{J_{t o t} \omega_{s}^{2}} \\
& -\frac{3}{2} \frac{\rho \pi R^{2}}{J_{t o t} \omega_{s}^{2}\left(1-\left\langle x_{2}\right\rangle\right)} C_{p}(\lambda, \theta)\left\langle x_{1}\right\rangle P_{11} \\
& -\frac{3}{2} \frac{\rho \pi R^{2}}{J_{t o t} \omega_{s}^{2}\left(1-\left\langle x_{2}\right\rangle\right)^{2}} C_{p}(\lambda, \theta)\left\langle x_{1}\right\rangle^{2} P_{12} \\
& -\frac{1}{2} \frac{\rho \pi R^{2}}{J_{t o t} \omega_{s}^{2}\left(1-\left\langle x_{2}\right\rangle\right)^{3}} C_{p}(\lambda, \theta)\left\langle x_{1}\right\rangle^{3} P_{22} \\
& A_{3}\left(\left\langle x_{t}\right\rangle, P_{t}\right)=\left\langle x_{2}\right\rangle\left\langle x_{4}\right\rangle \omega_{s}-\frac{1}{T_{0}^{\prime}}\left(\left\langle x_{3}\right\rangle+\left(X_{s}-X_{s}^{\prime}\right)\left\langle i_{q s}\right\rangle\right)+\omega_{s} P_{24} \\
& A_{4}\left(\left\langle x_{t}\right\rangle, P_{t}\right)=-\left\langle x_{2}\right\rangle\left\langle x_{3}\right\rangle \omega_{s}-\frac{1}{T_{0}^{\prime}}\left(\left\langle x_{4}\right\rangle-\left(X_{s}-X_{s}^{\prime}\right)\left\langle i_{d s}\right\rangle\right)-\omega_{s} P_{23}
\end{aligned}
$$

The diagonal elements of the matrix $B\left(\left\langle x_{t}\right\rangle, P_{t}\right)$ of Equation (7b) are the following:

$$
B_{11}\left(\left\langle x_{t}\right\rangle, P_{t}\right)=2 P_{11} \beta\left(-\frac{\sigma^{2}}{\left\langle x_{1}\right\rangle^{2}}-1\right)-6 P_{11}^{2} \beta \frac{\sigma^{2}}{\left\langle x_{1}\right\rangle^{4}}+2 \sigma^{2} \gamma^{2} \beta
$$




$$
\begin{aligned}
& B_{22}\left(\left\langle x_{t}\right\rangle, P_{t}\right)=-\frac{3 \rho \pi R^{2}}{J_{t o t} \omega_{s}^{2}\left(1-\left\langle x_{2}\right\rangle\right)} C_{p}(\lambda, \theta) P_{12}\left\langle x_{1}\right\rangle^{2} \\
& -\frac{\rho \pi R^{2}}{J_{t o t} \omega_{s}^{2}\left(1-\left\langle x_{2}\right\rangle\right)^{2}} C_{p}(\lambda, \theta) P_{22}\left\langle x_{1}\right\rangle^{3} \\
& -P_{12} P_{11} \frac{3 \rho \pi R^{2}}{J_{t o t} \omega_{s}^{2}\left(1-\left\langle x_{2}\right\rangle\right)} C_{p}(\lambda, \theta) \\
& -P_{12} P_{22} \frac{9 \rho \pi R^{2}}{J_{t o t} \omega_{s}^{2}\left(1-\left\langle x_{2}\right\rangle\right)^{3}} C_{p}(\lambda, \theta)\left\langle x_{1}\right\rangle^{2} \\
& -P_{12} P_{12} \frac{6 \rho \pi R^{2}}{J_{t o t} \omega_{s}^{2}\left(1-\left\langle x_{2}\right\rangle\right)^{2}}\left\langle x_{1}\right\rangle C_{p}(\lambda, \theta) \\
& -P_{22} P_{22} \frac{3 \rho \pi R^{2}}{J_{t o t} \omega_{s}^{2}\left(1-\left\langle x_{2}\right\rangle\right)^{4}} C_{p}(\lambda, \theta)\left\langle x_{1}\right\rangle^{3} \\
& -P_{11} P_{22} \frac{3 \rho \pi R^{2}}{J_{t o t} \omega_{s}^{2}\left(1-\left\langle x_{2}\right\rangle\right)^{2}} C_{p}(\lambda, \theta)\left\langle x_{1}\right\rangle \\
& B_{33}\left(\left\langle x_{t}\right\rangle, P_{t}\right)=2\left(P_{23}\left\langle x_{4}\right\rangle \omega_{s}+P_{34}\left\langle x_{2}\right\rangle \omega_{s}-\frac{1}{T_{0}^{\prime}} P_{33}\right) \\
& B_{44}\left(\left\langle x_{t}\right\rangle, P_{t}\right)=2\left(-P_{24}\left\langle x_{3}\right\rangle \omega_{s}-P_{34}\left\langle x_{2}\right\rangle \omega_{s}-\frac{1}{T_{0}^{\prime}} P_{44}\right)
\end{aligned}
$$

It is important to note that the terms $i_{d s}, i_{q s}, v_{d s}, v_{q s}, P_{e}, P_{m}$ are the system parameters of the stochastic system of this paper. The system parameter vector $\left(i_{d s}, i_{q s}, v_{d s}, v_{q s}, P_{e}, P_{m}\right)^{T}$ can be estimated using the algebraic Equations, (1e)-(1h), action of the expectation operator on random variables as well as Taylor expansions of non-linear functions with Gaussian assumptions. Note that the expectation operator is a linear operator. As a result of these, we get the following system of parameter estimation equations:

$$
\begin{aligned}
& \left\langle e_{d}^{\prime}\right\rangle=\left\langle x_{3}\right\rangle=-R_{s}\left\langle i_{d s}\right\rangle+X_{s}^{\prime}\left\langle i_{q s}\right\rangle+\left\langle v_{d s}\right\rangle \\
& \left\langle e_{q}^{\prime}\right\rangle=\left\langle x_{4}\right\rangle=-R_{s}\left\langle i_{q s}\right\rangle-X_{s}^{\prime}\left\langle i_{d s}\right\rangle+\left\langle v_{q s}\right\rangle \\
& \left\langle P_{e}\right\rangle=-\left\langle x_{3} i_{d s}\right\rangle-\left\langle x_{4} i_{q s}\right\rangle \\
& =-\left\langle x_{3}\right\rangle\left\langle i_{d s}\right\rangle-P_{x_{3} i_{d s}}-\left\langle x_{4}\right\rangle\left\langle i_{q s}\right\rangle-P_{x_{4} i_{q s}} \\
& =R_{s}\left\langle i_{d s}\right\rangle^{2}-X_{s}^{\prime}\left\langle i_{d s}\right\rangle\left\langle i_{q s}\right\rangle-\left\langle v_{d s}\right\rangle\left\langle i_{d s}\right\rangle-P_{x_{3} i_{d s}}
\end{aligned}
$$




$$
\begin{aligned}
& +R_{s}\left\langle i_{q s}\right\rangle^{2}+X_{s}^{\prime}\left\langle i_{d s}\right\rangle\left\langle i_{q s}\right\rangle-\left\langle v_{q s}\right\rangle\left\langle i_{q s}\right\rangle-P_{x_{4} i_{q s}} \\
& =R_{s}\left\langle i_{d s}\right\rangle^{2}-\left\langle v_{d s}\right\rangle\left\langle i_{d s}\right\rangle-P_{x_{3} i_{d s}}+R_{s}\left\langle i_{q s}\right\rangle^{2} \\
& -\left\langle v_{q s}\right\rangle\left\langle i_{q s}\right\rangle-P_{x_{4} i_{q s}} \\
& \left\langle P_{m}\right\rangle=\frac{1}{2} \rho \pi R^{2} C_{p}(\lambda, \theta)\left\langle x_{1}\right\rangle^{3} \approx \frac{1}{2} \rho \pi R^{2} C_{p}(\lambda, \theta)\left(\left\langle x_{1}\right\rangle^{3}+3 P_{11}\left\langle x_{1}\right\rangle\right)
\end{aligned}
$$

Note that notations must be carefully chosen for multi-dimensional systems. The electrical and mechanical power terms $P_{e}, P_{m}$ are different from the variance term notations $P_{x_{3} i d s}$ and $P_{x_{4} i s}$, where $P_{x_{3} i_{d s}}=\left\langle x_{3} i_{d s}\right\rangle-\left\langle x_{3}\right\rangle\left\langle i_{d s}\right\rangle, P_{x_{4} i_{q s}}=\left\langle x_{4} i_{q s}\right\rangle-\left\langle x_{4}\right\rangle\left\langle i_{q s}\right\rangle$. Equations (7a) and (7b) in combination with Equations (8a)-(8d) are the estimation results of the paper, which are not available in literature. That can be utilized for the state trajectory estimation of the stochastic system of the paper. More precisely, this paper can be treated as an application of an appealing non-linear stochastic method to an appealing stochastic system with random input signal. For understanding analysis of non-linear stochastic differential systems involving stochasticity in input signals as well as system parameters, Ku and Lin (1971) will be also useful.

Remark 1: Importantly, the wind generator power output is a stochastic power signal. Here, we explain an approach of Wächter et al. (2012), which exploits a stochastic model for the evolution of the wind generator power output in the Langevin setting. The stochastic power evolution for the fixed wind speed is:

$$
\frac{d P_{t}}{d t}=D^{1}\left(P_{t}, v_{t}\right)+\sqrt{D^{2}\left(P_{t}, v_{t}\right)} \Gamma_{t}
$$

where $P_{t}$ is a stochastic process, $D^{1}\left(P_{t}, v_{t}\right)$ is a drift term of the stochastic differential equation and $D^{2}\left(P_{t}, v_{t}\right)$ is the diffusion coefficient. This remark explains briefly the relation of the wind speed stochastic model to the stochastic power signal in the conditional expectation sense. The above can be recast in the Itô framework Kunita (2010), i.e.:

$$
d P_{t}=D^{1}\left(P_{t}, v_{t}\right) d t+\sqrt{D^{2}\left(P_{t}, v_{t}\right)} \Gamma_{t} d t=D^{1}\left(P_{t}, v_{t}\right) d t+\sqrt{D^{2}\left(P_{t}, v_{t}\right)} d B_{t}
$$

The Fokker-Planck equation for the above stochastic wind power evolution for the scalar case becomes:

$$
\frac{\partial p\left(P_{t} \mid v_{t}\right)}{\partial t}=-\frac{\partial D^{1}\left(P_{t}, v_{t}\right) p\left(P_{t} \mid v_{t}\right)}{\partial P_{t}}+\frac{1}{2} \frac{\partial^{2} D^{2}\left(P_{t}, v_{t}\right) p\left(P_{t} \mid v_{t}\right)}{\partial P_{t}^{2}}
$$


where the term $p\left(P_{t} \mid v_{t}\right)$ is a conditional probability density of the stochastic power for a given stochastic wind speed. The above Fokker-Planck equation is a convenient form to estimate the stochastic wind power. It is worth to note that the above relation holds under the condition that the stochastic power obeys the evolution equation stated in Wächter et al. (2012) for given wind speed, where the wind speed SDE obeys Equation (2a) of this discussion.

Remark 2: An alternative mathematical approach to achieve the estimation of the stochastic wind turbine-generator system is multi-dimensional Itô differential rules. Multi-dimensional Itô differential rules do not involve the notion of conditional probability density. Action of the conditional expectation operator on the stochastic evolution of the scalar function of the state vector leads to the conditional moment. In system theory, the scalar function has interpretation as the energy function, Lyapunov function. On the other hand, Fokker-Planck methods encompass the notion of conditional probability density, conditional expectation and conditional moment evolution equation. Thus, the Fokker-Planck approach of this paper is revealing greater insights into the stochastic system.

\section{NUMERICAL SIMULATIONS}

Here, we simulate the stochastic dynamics of the wind turbine-generator system by exploiting Equations (1a)-(1h) of the paper. That assume the structure of a vector stochastic differential equation. Subsequently, the random state trajectories are compared with the unperturbed state trajectories of the stochastic system considered here. To demonstrate the difference between the trajectories of random state and unperturbed state, we exploit two different sets of initial data and system parameters. Notably, the first set of numerical simulation data is utilized for numerical experimentations for a relatively low power rating machine. On the other hand, the second set of data is intended for numerical experimentations for a larger power rating machine. Furthermore, the strength of stochastic perturbations is same in the both sets of data. This criterion is adopted for the numerical experimentation to reveal the stochastic influence on lower and larger power rating machines. Since the noise-influenced wind turbine-generator system involves a multi-dimensional stochastic differential equation structure, numerical experimentations become quite difficult. However, this paper attempts to accomplish the numerical experimentations for two different sets of data. First, we demonstrate numerical simulations for the random state trajectories and subsequently, the estimated state trajectories. Here, we utilize the following first set of initial data and system parameters for the machine rating of $4.718 \mathrm{MW}$ :

$$
\begin{aligned}
& V=6600 \mathrm{~V}, \mathrm{~F}=60 \mathrm{~Hz}, P_{r}=3, R_{s}=0.04530 \Omega, R_{r}=0.08015 \Omega, X_{r}=1.1325 \Omega \\
& X_{s}=42.5753 \Omega, X_{s}^{\prime}=1.8779 \Omega, R=63 \mathrm{~m}, x_{4}(0)=1108.33 \mathrm{~V}, T_{0}^{\prime}=1.4215 \mathrm{sec} \\
& J_{t o t} \omega_{s}^{2}=100.16 \mathrm{~kg} \mathrm{~m} \mathrm{rad}^{2} / \mathrm{sec}^{2}, \rho=1.225 \mathrm{~kg} / \mathrm{m}^{3} \\
& x_{1}(0)=12 \mathrm{~m} / \mathrm{s}, x_{2}(0)=-0.008845, x_{3}(0)=5040.11 \mathrm{~V} \\
& i_{d s}(0)=-585.37 \mathrm{~A}, i_{q s}(0)=-199.84 \mathrm{~A}, P_{e}=3171817.85 \mathrm{~W} \\
& \gamma=1, \sigma=10 \mathrm{~m} / \mathrm{s}, \beta=2 \mathrm{sec}^{-1}, C_{p}(\lambda, \theta)=0.3563
\end{aligned}
$$

The set of system parameters of a smaller power rating machine can be found in Li et al. (2006) in per unit interpretation as well. For more clarity, we have utilized the dimensional interpretation in lieu of the dimensionless per unit interpretation. Note that the solid line (-) trajectories of Figures 
3-6 denote the state trajectories resulting from the noise-free dynamics of the stochastic system considered here. The stochastic correction term of Equation (2a) is ignored. On the other hand, the dotted line (--) trajectories of Figures 3-6 denote the stochastically-influenced state trajectories of the wind turbine-generator system. That are the consequence of Equations (2a)-(2d) of the paper. The difference between the solid line and dotted line trajectories, Figures 3-6, suggests that the stochastic influence is considerable in three states, slip, the $d$-axis voltage behind transient impedance and the $q$-axis voltage behind transient impedance. It is important to observe that the noise-free state trajectories follow the noise-influenced state trajectories. However, the stochastic correction term introduces modifications into the random state trajectories.

Here, we simulate the random state dynamics of a larger power rating machine. The second set of initial data and system parameters for numerical simulations of the machine rating of $10 \mathrm{MW}$, a larger power rating machine, is the following:

$$
\begin{aligned}
& V=8076.55 \mathrm{~V}, \mathrm{~F}=60 \mathrm{~Hz}, P_{r}=3, R_{s}=0.03921 \Omega, R_{r}=0.01245 \Omega \\
& X_{r}=0.3685 \Omega, X_{s}=38.0484 \Omega, X_{s}^{\prime}=0.7533 \Omega, R=85 \mathrm{~m}
\end{aligned}
$$

Figure 3. A comparison between unperturbed and stochastic trajectories of the state $x 1$

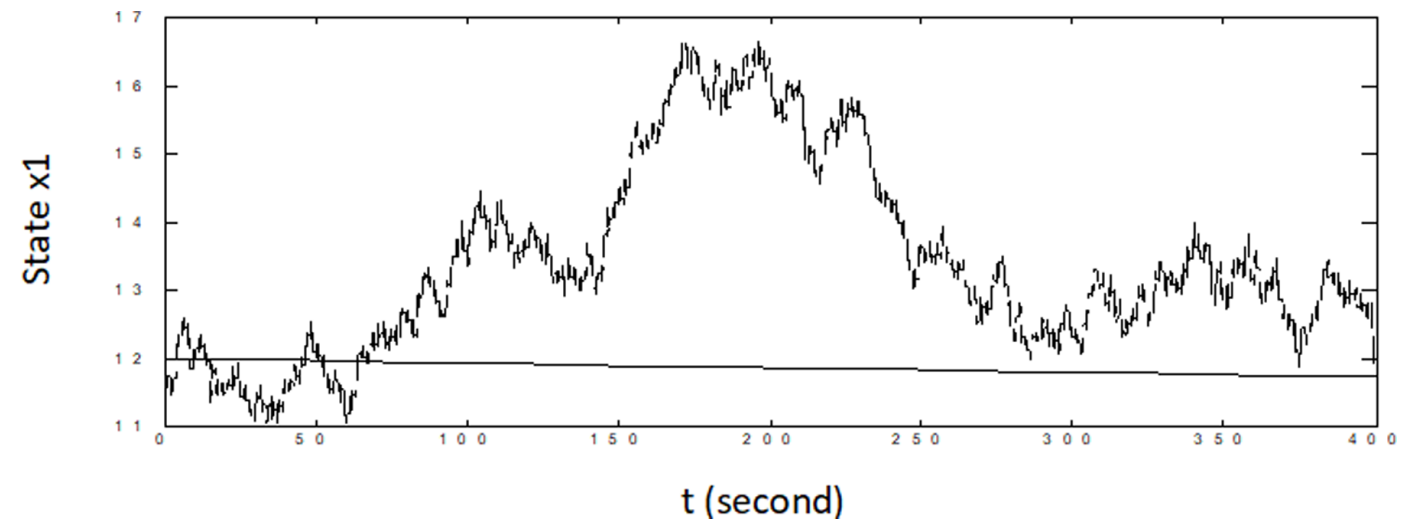

Figure 4. A comparison between unperturbed and stochastic trajectories of the state $\mathrm{x} 2$

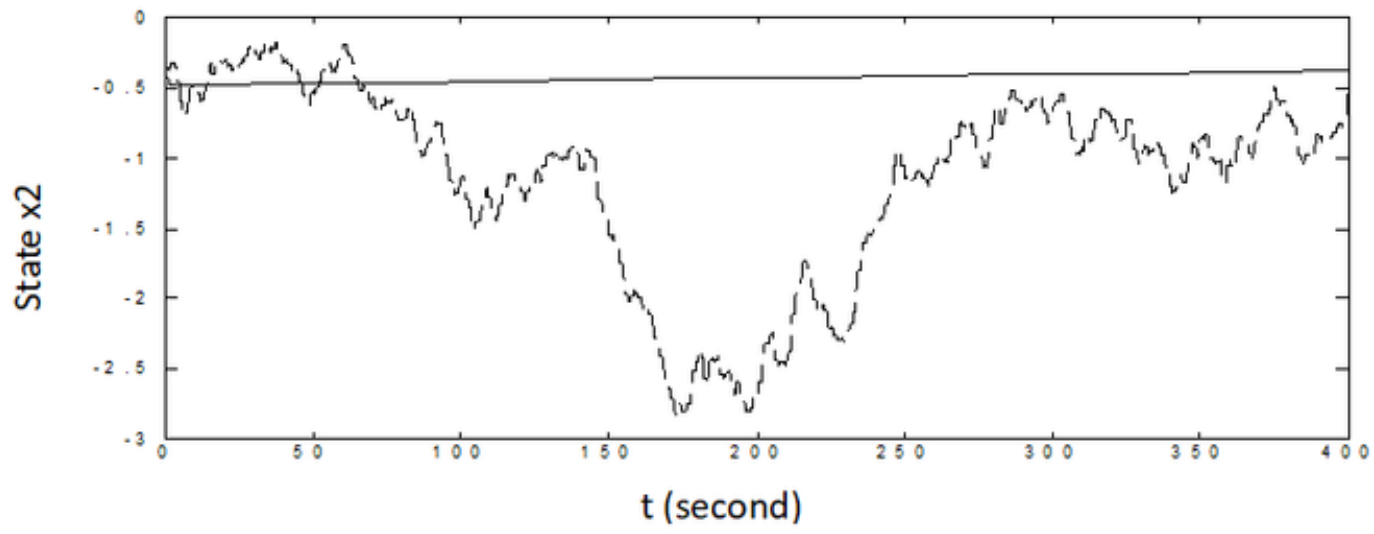


Figure 5. A comparison between unperturbed and stochastic trajectories of the state $x 3$

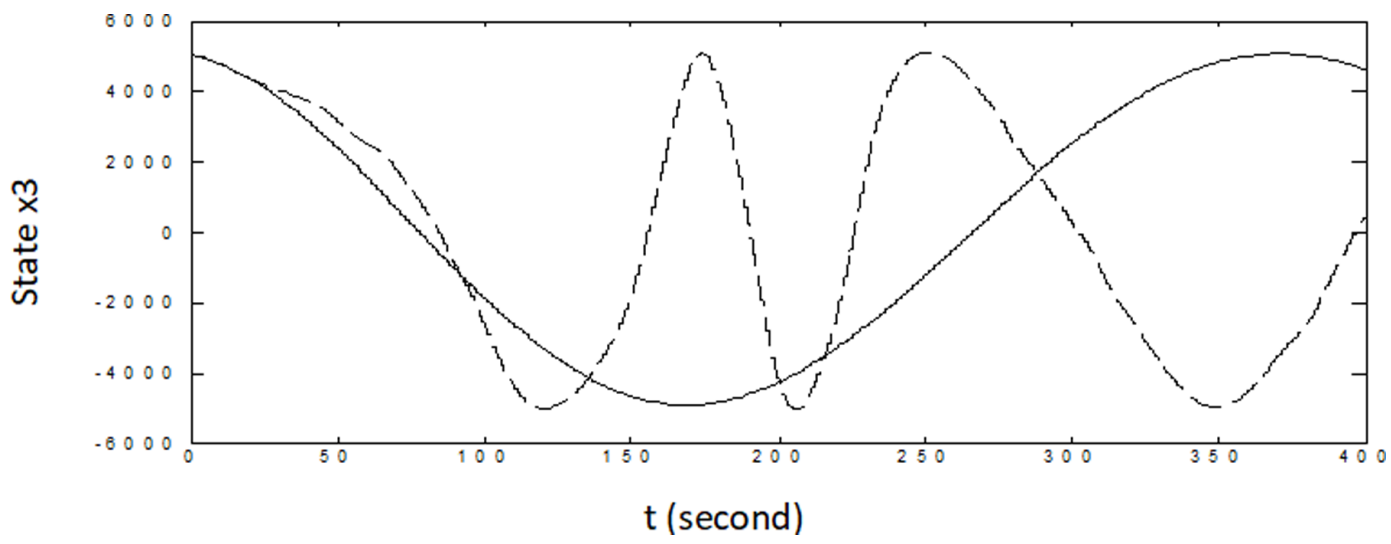

Figure 6. A comparison between unperturbed and stochastic trajectories of the state $x 4$

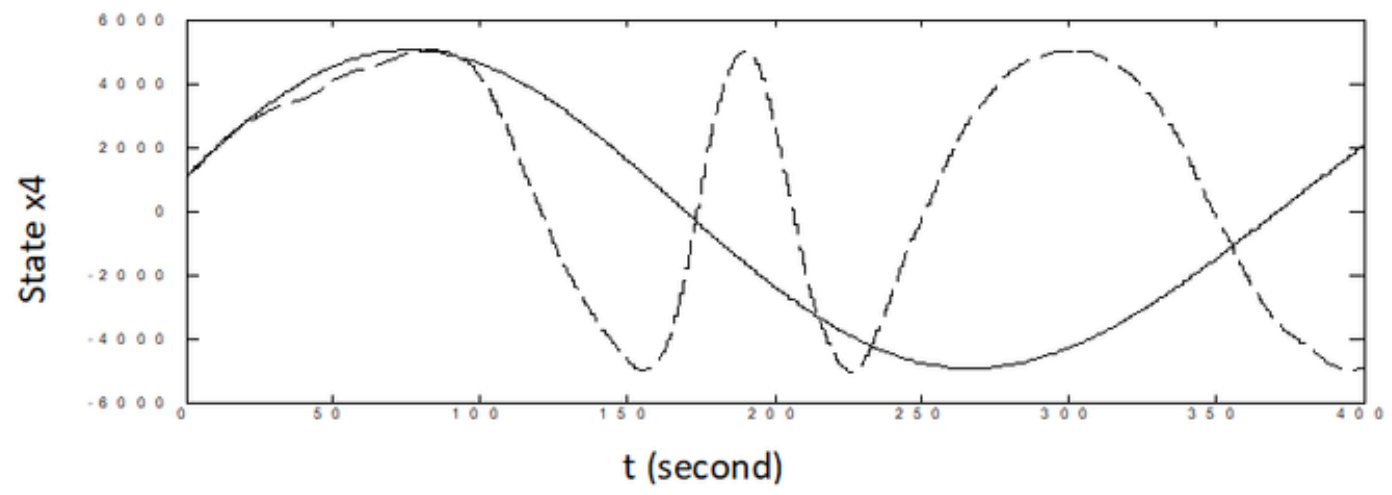

$J_{\text {tot }} \omega_{s}^{2}=1000.16 \mathrm{~kg} \mathrm{~m} \mathrm{rad}^{2} / \mathrm{sec}^{2}, \rho=1.225 \mathrm{~kg} / \mathrm{m}^{3}, \gamma=1$

$\sigma=10 \mathrm{~m} / \mathrm{s}, \beta=2 \sec ^{-1}, C_{p}(\lambda, \theta)=0.4164$

$x_{1}(0)=12 \mathrm{~m} / \mathrm{s}, x_{2}(0)=-0.0096, x_{3}(0)=6459.12 \mathrm{~V}, x_{4}(0)=788.49 \mathrm{~V}, T_{0}^{\prime}=8.1064 \mathrm{sec}$

$i_{d s}=-1034.56 A, i_{q s}=-233.52 A, P_{e}=6866475.37 \mathrm{~W}$

The set of system parameters of the larger power rating machine in per unit interpretation can be found in Tamura (2012), see Li et al. (2006) as well. Similar to the first set of data, we adopt the dimensional interpretation of system parameters as well. Note that the solid line (-) of Figures 7-10 denotes the state trajectories resulting from the noise-free dynamics of the stochastic system considered here in which the stochastic correction term of Equations (2a)-(2d) is ignored. On the other hand, the dotted line (--) trajectories of Figures 7-10 denote the stochastically-influenced state trajectories of the wind turbine-generator system. Similar qualitative characteristics of the random state vector of the wind turbine-generator system are demonstrated using the second set of initial data and system parameters as well. Numerical simulations demonstrated in Figures 3-6 and Figures 7-10 suggest that stochastic considerations are imperative for larger and lower power rating wind turbine-generator systems. 
Figure 7. A comparison between unperturbed and stochastic trajectories of the state $\mathrm{x} 1$

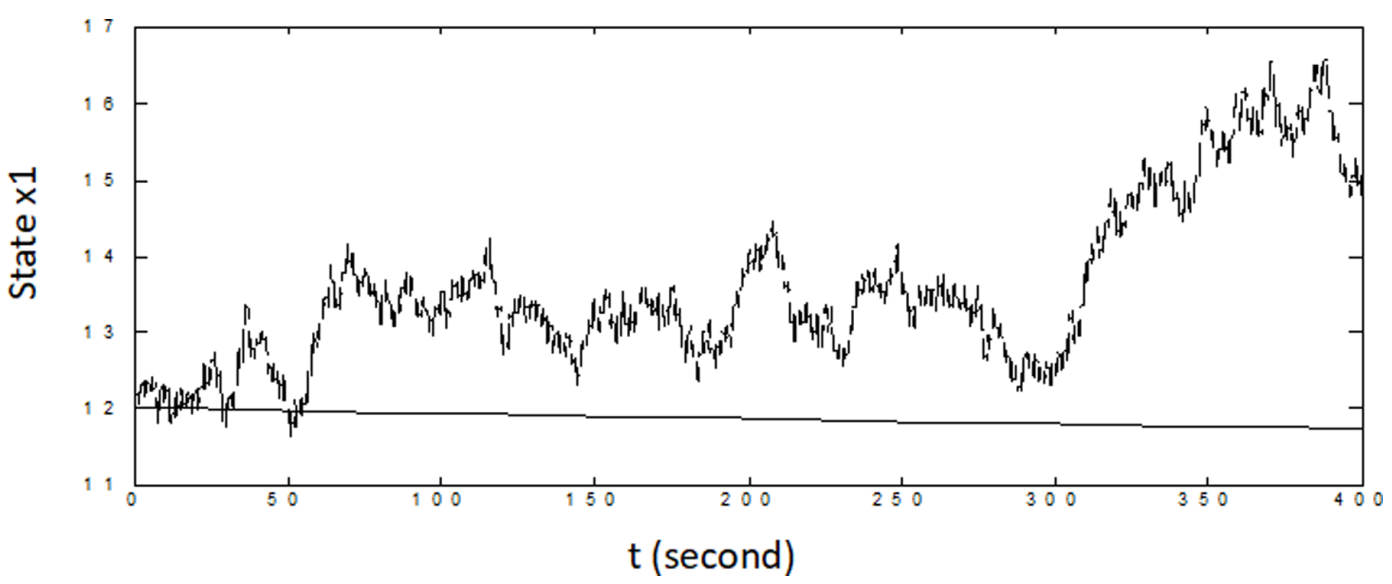

The estimated state trajectories are compared with unperturbed state trajectories. The dotted line (--) trajectories of Figures 11-13 illustrate the estimated state trajectories. On the other hand, the solid line (-) trajectories denote the unperturbed trajectories. The estimated state trajectories are bounded continuous that demonstrate the effectiveness of the state estimation as well as the parameter estimation equations of the paper. The estimation equations of the paper, (6a)-(6b), have ability to preserve qualitative characteristics of linear terms, square non-linearity as well as cubic non-linearity of nonlinear stochastic differential systems completely. That preserve some of the qualitative characteristics of higher-order non-linearities as well. Note that the state estimation equations coupled with the parameter estimation equations, Equations (7a)-(7b) and Equations (8a)-(8d), are specific case of Equations (6a)-(6b). Thus, 'the state estimation equations coupled with the parameter estimation equations' are effective for state trajectory estimations of the stochastic system of the paper.

Here, we explain succinctly the qualitative characteristics of the state $x_{2}$ of the stochastic system of the paper. Note that the state $x_{2}$ denotes the stochastic evolution of the slip of the wind turbinegenerator system. For the given set of data and system parameters, the most probable slip trajectory of the induction machine is the bounded continuous. This confirms the general theory of induction machines that the slip of the machine in the generating mode takes finite negative values on the real

Figure 8. A comparison between unperturbed and stochastic trajectories of the state $\mathrm{x} 2$

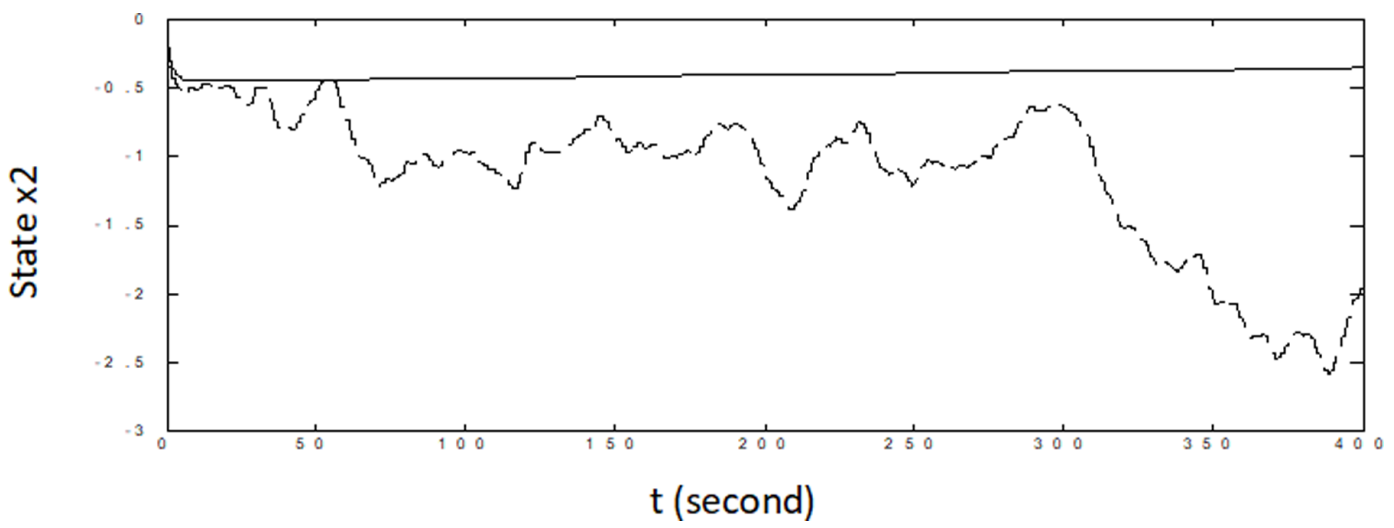


Figure 9. A comparison between unperturbed and stochastic trajectories of the state $x 3$

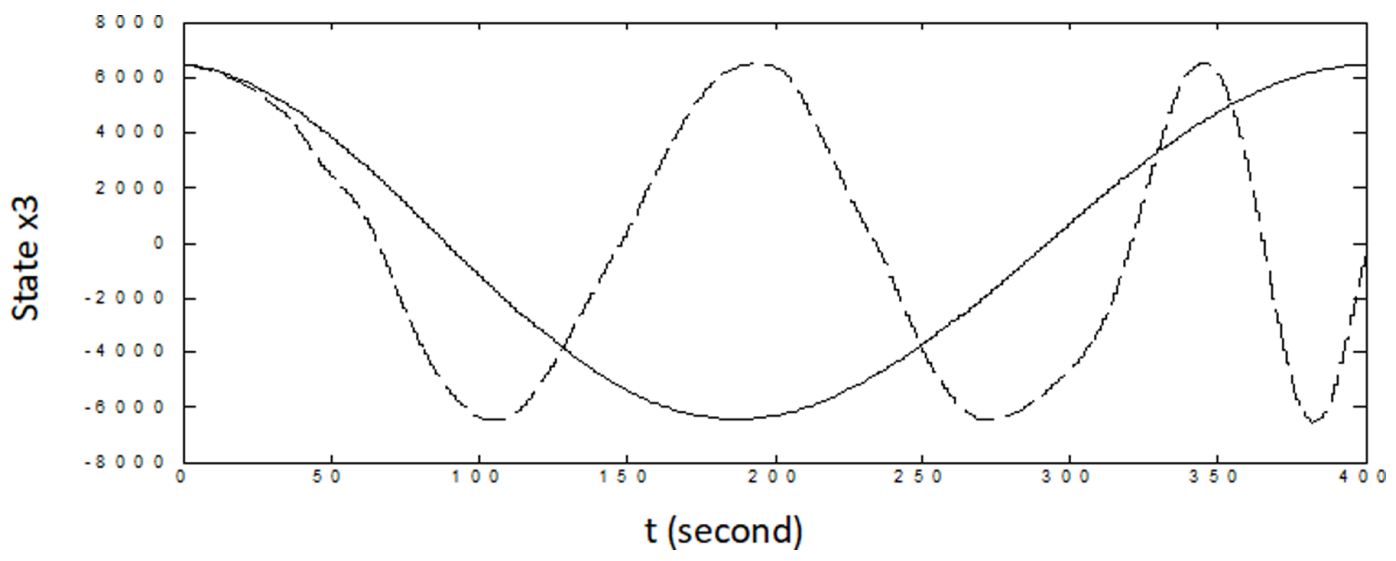

Figure 10. A comparison between unperturbed and stochastic trajectories of the state $x 4$

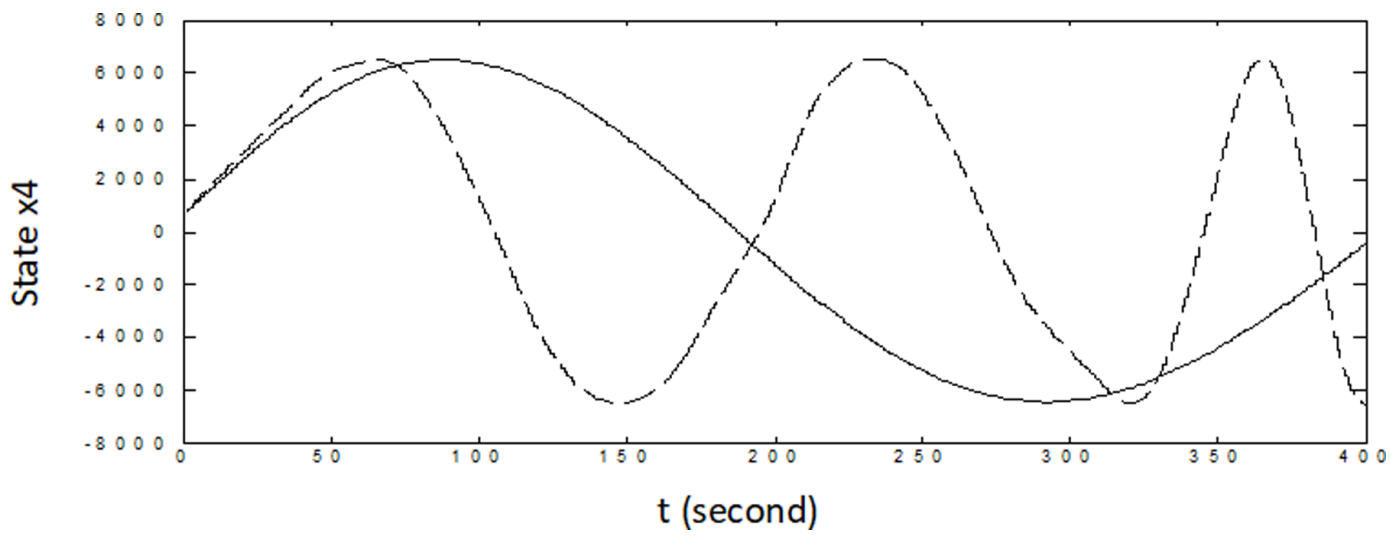

Figure 11. A comparison between unperturbed and estimated trajectories of the state $\mathrm{x} 2$

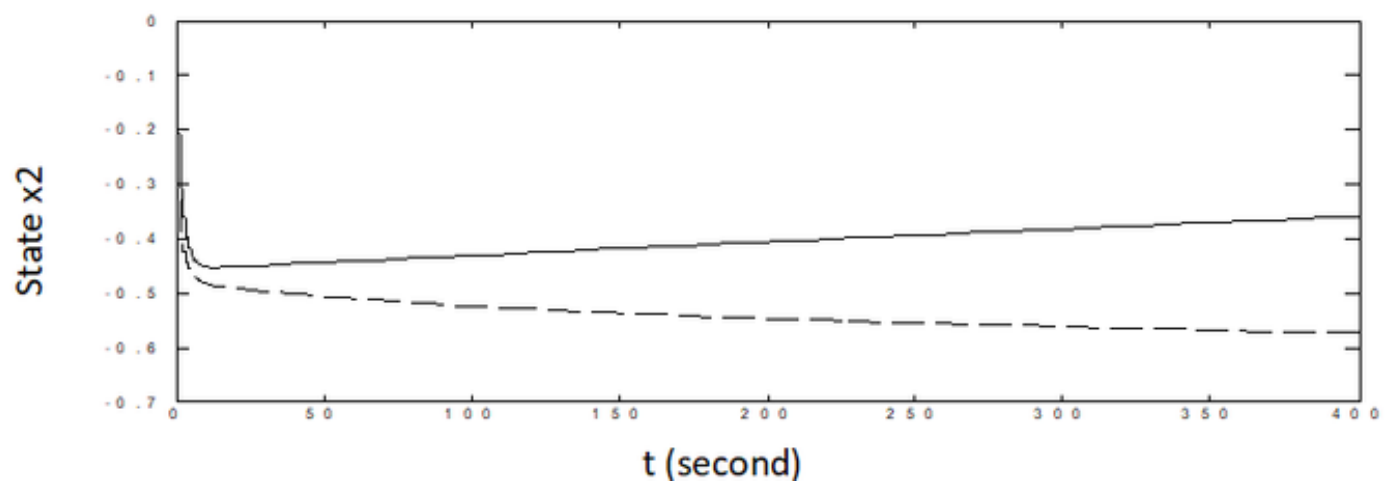




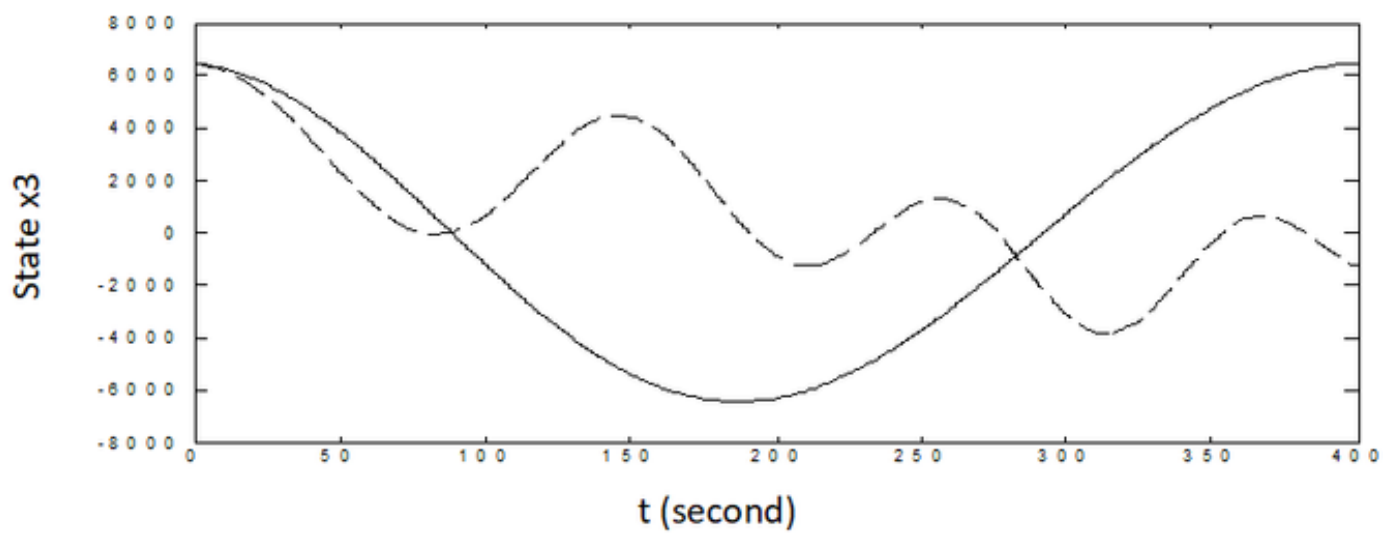

Figure 13. A comparison between unperturbed and estimated trajectories of the state $x 4$

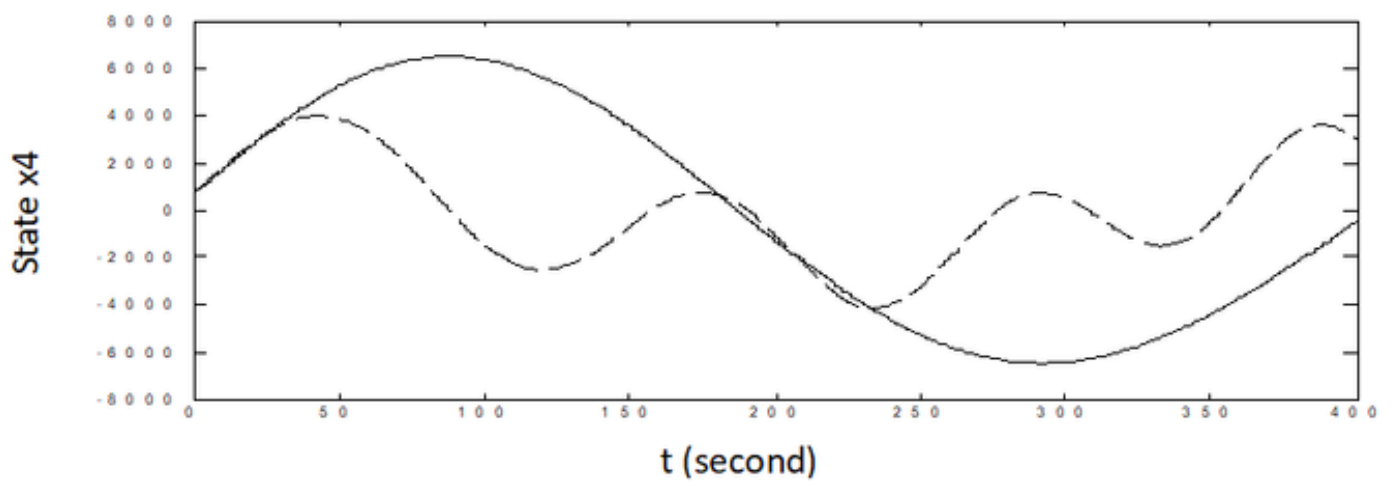

line. Thus, the numerical simulation suggests the efficacy of the conditional moment equations of the paper. The estimated trajectories of the voltage stochastic states of the machine can be explained using similar arguments as well. Thus, further discussions are omitted.

\section{CONCLUSION}

The main achievement of this paper is to develop a Markovian stochastic dynamics of the wind turbine-generator system. The Markovian stochastic dynamics is attributed to small scale atmospheric turbulence influence on the wind turbine-generator system. Subsequently, we have accomplished a Fokker-Planck analysis of the stochastic wind energy system as well, which is not available in literature. The numerical simulations demonstrated in Figures 3-6 as well as Figures 7-10 reveal that the differences between the unperturbed and perturbed state trajectories become considerable for lower as well as larger power rating machines. Thus, this paper suggests that stochasticity considerations are imperative in lieu of deterministic considerations. This paper confirms the universality of small scale turbulence (Schumacher et al. 2014) by embedding the qualitative characteristics of the small-scale turbulence in the wind speed evolution equation via the Itô stochastic correction term.

More precisely, we have accomplished the noise analysis of the wind turbine-generator system from the estimation-theoretic viewpoint by exploiting the Fokker-Planck approach of stochastic processes. The Fokker-Planck model is an advanced, a refined mathematical method of stochastic 
processes. That has found its striking applications in deriving estimation algorithms, stability conditions and control laws as well as achieving stochastic bifurcation analysis of stochastic differential systems. The Fokker-Planck method accounts for stochastic correction terms, on the other hand, classical methods do not.

The estimation equations for the state vector of the wind turbine-generator system, Equations (7a)-(7b) coupled with Equations (8a)-(8d), will be useful for the control of the state vector $x_{t}=\left(v_{t}, s, e_{d}^{\prime}, e_{q}^{\prime}\right)^{T}$ of the wind turbine-generator system as well.

For these reasons, it is believed that this paper will have lasting influence not only in power systems dynamics literature, that will be of interest to turbulence dynamists pursuing their research in non-linear vector SDEs arising from diverse fields, e.g. fluctuating aerodynamics, and underwater vehicle dynamics. 


\section{REFERENCES}

Alain, K. S. T., Romanic, K., \& Bertrand, F. H. (2017). Dynamics and Improved Robust Adaptive Control Strategy for the Finite Time Synchronization of Uncertain Nonlinear Systems. International Journal of System Dynamics Applications, 6(4), 34-62. doi:10.4018/IJSDA.2017100103

Balanathan, R., Pahalawaththa, N. C., \& Annakkage, U. D. (2002). Modelling Induction Motor Loads for Voltage Stability Analysis. Electrical Power \& Energy Systems, 24(6), 469-480. doi:10.1016/S0142-0615(01)00059-X

Bierbaum, M. M., Joseph, R. I., Fry, R. L., \& Nelson, J. B. (2002). A Fokker-Planck Model for a Two-Body Problem. Proceedings of AIP Conference, 617, 340-371. doi:10.1063/1.1477058

Chow, S. N., \& Zhou, H. M. (2007). An Analysis of Phase Noise and Fokker-Planck Equations. Journal of Differential Equations, 234(2), 391-411. doi:10.1016/j.jde.2006.11.015

Diaconis, P. (1987). Application of the Method of Moments in Probability and Statistics. In H.J. Landau (Eds.), Moments in Mathematics - Proceedings of Symposia in Applied Mathematics (pp. 125-142). American Mathematical Society.

Dusonchet, L., Massaro, F., \& Telaretti, E. (2007). Transient Stability Simulation of a Fixed Speed Wind Turbine by Matlab/Simulink. In Proceedings of the International Conference on Clean Electric Power, Capri, Italy (pp. 651-655). Academic Press. doi:10.1109/ICCEP.2007.384308

Feijóo, A., Cidrás, J., \& Carrillo, C. (2000). A third-order model for doubly-fed induction machine. Electric Power Systems Research, 56(1), 121-127. doi:10.1016/S0378-7796(00)00103-6

Friedrich, R., \& Peinke, J. (1997). Description of a turbulent cascade by a Fokker-Planck equation. Physical Review Letters, 78(5), 863-866. doi:10.1103/PhysRevLett.78.863

Friedrich, R., Peinke, J., Sahimi, M., \& Tabar, M. R. R. (2011). Approaching complexity by stochastic methods: From biological systems to turbulence. Physics Reports, 506(5), 87-162. doi:10.1016/j.physrep.2011.05.003

Fu, D., \& Xing, Y. (2009). Study on Linear Dynamic and Analysis of Operating Characteristics of High-Power VSCF Wind Energy Conversion System. In Proceedings of the World Non-grid-connected Wind Power and Energy Conference (WNWEC), Nanjing, China. Academic Press. doi:10.1109/WNWEC.2009.5335766

Ghabi, J., Rhif, A., \& Vaidyanathan, S. (2018). Discrete time sliding mode control scheme for nonlinear systems with bounded uncertainties. International Journal of System Dynamics Applications, 7(2), 15-33. doi:10.4018/ IJSDA.2018040102

Greets, S., Stones, J., Driesen, J., Belmans, R., \& Hirsch, C. (2005). Entire Wind-to-Power Representation of a Wind Turbine Unit in Electrical Power System Studies. In Proceedings of the 18th International Conference on Electricity Distribution, Turin, Italy. Academic Press. doi:10.1049/cp:20051276

Jazwinski, A. H. (1970). Stochastic Processes and Filtering Theory. New York: Academic Press.

Karatzas, I., \& Shreve, S. E. (1988). Brownian Motion and Stochastic Calculus. New York: Springer. doi:10.1007/978-1-4684-0302-2

Karlsen, H. C. (2006). Statistics of Wave Induced Nonlinear Loads and Responses [Ph.D. Dissertation]. Norwegian University of Science and Technology, Trondheim, Norway.

Kolmogorov, A. N. (1941). The local structure of turbulence in an incompressible viscous fluid for very large Reynolds numbers. Doklady Akademii Nauk SSSR, 30, 301-305.

Kolmogorov, A. N. (1962). A refinement of previous hypotheses concerning the local structure of turbulence in a viscous incompressible fluid at high Reynolds number. Journal of Fluid Mechanics, 13(1), 82-85. doi:10.1017/ S0022112062000518

Ku, Y. H., \& Lin, T. S. (1971). Analysis of Nonlinear Systems with Stochastic Input and Stochastic Parameters. Journal of the Franklin Institute, 292(5), 313-331. doi:10.1016/0016-0032(71)90060-3

Kundur, P. (1994). Power System Stability and Control. New York, US: McGraw-Hill. 
Kunita, H. (2010). Itô Stochastic Calculus: Its Surprising Power for Applications. Stochastic Processes and Their Applications, 120(5), 622-652. doi:10.1016/j.spa.2010.01.013

Kuo, H. H. (2005). White Noise Stochastic Integration, Stochastic Analysis Classical and Quantum. In Eproceedings, World Scientific (pp. 57-71). Academic Press.

Kushner, H. J. (1967). Stochastic Stability and Control. New York: Academic Press.

Ledesma, P., Usaola, J., \& Rodríguez, J. L. (2003). Transient Stability of a Fixed Speed Wind Farm. Renewable Energy, 28(9), 1341-1355. doi:10.1016/S0960-1481(02)00251-3

Li, H., \& Chen, Z. (2008). Overview of Different Wind Generator Systems and Their Comparisons. IET Renewable Power Generation, 2(2), 123-138. doi:10.1049/iet-rpg:20070044

Li, H., Chen, Z., \& Polinder, H. (2006). Research Report on Numerical Evaluation of Various Variable Speed Wind Generator Systems. Up Wind.

Martins, M., Perdana, A., Ledesma, P., Agneholm, E., \& Carlson, O. (2007). Validation of Fixed Speed Wind Turbine Dynamic Models with Measured Data. Renewable Energy, 32(8), 1301-1316. doi:10.1016/j. renene.2006.06.007

Milan, P., Wächter, M., \& Peinke, J. (2013). Turbulent character of wind energy. Physical Review Letters, 110(13).

Moysis, L., Azar, A. T., Kafetzis, I., Tsiaousis, M., \& Charalampidis, N. (2017). Introduction to Control Systems Design Using Matlab. International Journal of System Dynamics Applications, 6(3), 130-170. doi:10.4018/ IJSDA.2017070107

Mücke, T., Wächter, M., Milan, P., \& Peinke, J. (2015). Langevin power curve analysis for numerical wind energy converter models with new insights on high frequency power performance. Wind Energy (Chichester, England), 18(11), 1953-1971. doi:10.1002/we.1799

Mumford, D. (2000). The Dawning of the Age of Stochasticity, Mathematics Frontiers and Perspectives. American Mathematical Society.

Nichita, C., Luca, D., Dakyo, B., \& Ceanga, E. (2002). Large Band Simulation of the Wind Speed for Real Time Wind Turbine Simulators. IEEE Transactions on Energy Conversion, 17(4), 523-529. doi:10.1109/ TEC.2002.805216

Patel, H. G., \& Sharma, S. N. (2014). Third-order Continuous-Discrete Filtering for a Nonlinear Dynamic System. The ASME Transactions, Journal of Computational and Nonlinear Dynamics, 9(3). doi:.10.1115/1.4026064

Pedrizzetti, G., \& Novikov, E. A. (1994). On Markov modelling of turbulence. Journal of Fluid Mechanics, 280, 69-93. doi:10.1017/S0022112094002855

Pintea, A., Wang, H., Christov, N., Borne, P., \& Popescu, D. (2011). Modelling and Power Regulation of Horizontal Variable Speed Wind Turbines. Studies in Informatics and Control, 20(3), 305-312. doi:10.24846/v20i3y201111

Popović, D. H., Hiskens, I. A., \& Hill, D. J. (1998). Stability Analysis of Induction Motor Networks. Electrical Power \& Energy Systems, 20(7), 475-487. doi:10.1016/S0142-0615(98)00011-8

Renner, Ch., Peinke, J., \& Friedrich, R. (2001). Experimental Indications for Markov Properties of Small Scale Turbulence. Journal of Fluid Mechanics, 433, 383-409. doi:10.1017/S0022112001003597

Risken, H. (1984). The Fokker-Planck Equation, Methods of Solution and Applications. Berlin, Germany: Springer-Verlag. doi:10.1007/978-3-642-96807-5

Salima, M., Ahras, S., Loubna, A., \& Riad, T. (2018). A Global Stability of Linearizing Control of Induction Motor for PV Water Pumping Application. International Journal of System Dynamics Applications, 7(3), 31-56. doi:10.4018/IJSDA.2018070102

Schumacher, J., Scheel, J. D., Krasnov, D., Donzis, D. A., Yakhot, V., \& Sreenivasan, K. R. (2014). Small-Scale Universality in Fluid Turbulence. Proceedings of the National Academy of Sciences of the United States of America, 111(30), 10961-10965. doi:10.1073/pnas.1410791111 PMID:25024175

Sharma, S. N. (2008). A Kolmogorov-Fokker-Planck Approach for a Stochastic Duffing-van der Pol System. Differential Equations and Dynamical Systems, 16(4), 351-377. 
Sinai, Y. A. G. (1981). The Stochasticity of Dynamical Systems. Selecta Math. Soviet, 1, 100-119.

Slootweg, J. G., Polinder, H., \& Kling, W. L. (2003). Representing Wind Turbine Electrical Generating Systems in Fundamental Frequency Simulations. IEEE Transactions on Energy Conversion, 18(4), 516-524. doi:10.1109/ TEC.2003.816593

Sorensen, P., Hansen, A. D., Adrè, P., \& Rosas, C. (2002). Wind Models for Simulation of Power Fluctuations from Wind Farms. Journal of Wind Engineering and Industrial Aerodynamics, 90(12-15), 1381-1402. doi:10.1016/ S0167-6105(02)00260-X

Taha Hussien, H. A., Ammar, M. E., \& Moustafa Hassan, M. A. (2017). Three Phase Induction Motor's Stator Turns Fault Analysis Based on Artificial Intelligence. International Journal of System Dynamics Applications, 6(3), 1-19. doi:10.4018/IJSDA.2017070101

Tamura, J. (2012). Wind Energy Conversion Systems, Green Energy and Technology. London, UK: SpringerVerlag.

Wächter, M., Heißelmann, H., Hölling, M., Morales, A., Milan, P., Mücke, T., \& Rinn, P. et al. (2012). The turbulent nature of the atmospheric boundary layer and its impact on the wind energy conversion process. Journal of Turbulence, 13, 1-13. doi:10.1080/14685248.2012.696118

Wang, K., \& Crow, M. L. (2012). Fokker- Planck Equation Application to Analysis of a Simplified Wind Turbine Model. In Proceedings of the North American Power Symposium (NAPS), Champaign, IL. Academic Press.

Wu, B., Lang, Y., Zargari, N., \& Kouro, S. (2011). Power Conversion and Control of Wind Energy Systems. New Jersey: John Wiley \& Sons, Inc. doi:10.1002/9781118029008 


\section{APPENDIX}

Equation (4) describes the Fokker-Planck equation of the Itô stochastic differential system. The alternative interpretation of the Fokker-Planck equation in the matrix-vector format is:

$$
d p=\left(-\operatorname{tr} \frac{\partial f(x, t) p}{\partial x}+\frac{1}{2} \operatorname{tr} \frac{\partial^{2}\left(G G^{T}\right)(x, t) p}{\partial x \partial x^{T}}\right) d t
$$

The Fokker-Planck operator $L($.$) that acts on the conditional probability density for Markov processes$ can be stated alternatively as:

$$
L(.)=-\operatorname{tr} \frac{\partial f(x, t)(.)}{\partial x}+\frac{1}{2} \operatorname{tr} \frac{\partial^{2}\left(G G^{T}\right)(x, t)(.)}{\partial x \partial x^{T}}
$$

Importantly, the Fokker-Planck operator has the adjoint property, i.e. the Kolmogorov backward operator is the adjoint of the Fokker-Planck operator. The Kolmogorov backward operator $L^{\prime}($.$) for$ the Itô stochastic differential system is:

$$
\begin{aligned}
& L^{\prime}(.)=f^{T}(x, t) \frac{\partial(.)}{\partial x}+\frac{1}{2} \operatorname{tr}\left(\left(G G^{T}\right)(x, t) \frac{\partial^{2}(.)}{\partial x \partial x^{T}}\right) \\
& =\sum_{i} f_{i}(x, t) \frac{\partial(.)}{\partial x_{i}}+\frac{1}{2} \sum_{i, j}\left(G G^{T}\right)_{i j}(x, t) \frac{\partial^{2}(.)}{\partial x_{i} \partial x_{j}}
\end{aligned}
$$

Alternative interpretation of the backward operator of Equation (A1) is:

$$
L^{\prime}(.)=\left\langle f, \frac{\partial(.)}{\partial x}\right\rangle+\frac{1}{2} \operatorname{tr}\left(\left(G G^{T}\right) \frac{\partial^{2}(.)}{\partial x \partial x^{T}}\right)=\operatorname{tr}\left(f \frac{\partial(.)}{\partial x^{T}}\right)+\frac{1}{2} \operatorname{tr}\left(\left(G G^{T}\right)(x, t) \frac{\partial^{2}(.)}{\partial x \partial x^{T}}\right)
$$

Making the use of the Kolmogorov forward operator, backward operator as well as the definition of the evolution $d\left\langle\varphi\left(x_{t}\right)\right\rangle$ of conditional moment (Diaconis, 1987), we get:

$$
d\left\langle\varphi\left(x_{t}\right)\right\rangle=\left(\left\langle f^{T} \frac{\partial \varphi\left(x_{t}\right)}{\partial x_{t}}\right\rangle+\frac{1}{2}\left\langle\operatorname{tr}\left(\left(G G^{T}\right)\left(x_{t}, t\right) \frac{\partial^{2} \varphi\left(x_{t}\right)}{\partial x_{t} \partial x_{t}^{T}}\right)\right\rangle d t\right.
$$

After considering $\varphi\left(x_{t}\right)=x_{i}$ as well as $\varphi\left(x_{t}\right)=x_{i} x_{j}$, we get the exact conditional mean and conditional variance evolutions, i.e.:

$$
d\left\langle x_{i}\right\rangle=\left\langle f_{i}\left(x_{t}, t\right)\right\rangle d t
$$




$$
d P_{i j}=\left(\left\langle x_{i} f_{j}\right\rangle-\left\langle x_{i}\right\rangle\left\langle f_{j}\right\rangle+\left\langle f_{i} x_{j}\right\rangle-\left\langle f_{i}\right\rangle\left\langle x_{j}\right\rangle+\left\langle\left(G G^{T}\right)_{i, j}\left(x_{t}, t\right)\right\rangle\right) d t
$$

The Appendix Equations (A2a) and (A2b) can be regarded as a system of two coupled first-order exact differential equations. After considering the high-order partials of the system non-linearity $f_{i}\left(x_{t}, t\right)$ and the diffusion coefficient $\left(G G^{T}\right)_{i j}\left(x_{t}, t\right)$ as well as Gaussian assumptions, we are led to Equations (A2a)-(A2b) of the paper, i.e.:

$$
\begin{aligned}
& d\left\langle x_{i}(t)\right\rangle=\left(f_{i}\left(\left\langle x_{t}\right\rangle, t\right)+\frac{1}{2} \sum_{p, q} P_{p q} \frac{\partial^{2} f_{i}\left(\left\langle x_{t}\right\rangle, t\right)}{\partial\left\langle x_{p}\right\rangle \partial\left\langle x_{q}\right\rangle}\right) d t \\
& d P_{i j}=\left(\begin{array}{l}
\sum_{p} P_{i p} \frac{\partial f_{j}\left(\left\langle x_{t}\right\rangle, t\right)}{\partial\left\langle x_{p}\right\rangle}+\sum_{p} P_{j p} \frac{\partial f_{i}\left(\left\langle x_{t}\right\rangle, t\right)}{\partial\left\langle x_{p}\right\rangle} \\
+\frac{1}{2} \sum_{p, q, r} P_{i p} P_{q r} \frac{\partial^{3} f_{j}\left(\left\langle x_{t}\right\rangle, t\right)}{\partial\left\langle x_{p}\right\rangle \partial\left\langle x_{q}\right\rangle \partial\left\langle x_{r}\right\rangle}+\frac{1}{2} \sum_{p, q, r} P_{j p} P_{q r} \frac{\partial^{3} f_{i}\left(\left\langle x_{t}\right\rangle, t\right)}{\partial\left\langle x_{p}\right\rangle \partial\left\langle x_{q}\right\rangle \partial\left\langle x_{r}\right\rangle} \\
+\left(G G^{T}\right)_{i j}\left(\left\langle x_{t}\right\rangle, t\right)+\frac{1}{2} \sum_{p, q} P_{p q} \frac{\partial^{2}\left(G G^{T}\right)_{i j}\left(\left\langle x_{t}\right\rangle, t\right)}{\partial\left\langle x_{p}\right\rangle \partial\left\langle x_{q}\right\rangle}
\end{array}\right) d t
\end{aligned}
$$

Q. E. D

A good discussion about the above system of equations can be found in Sharma (2008) as well. That introduces different notations and interpretations.

Remark 3: The K L expansion has proven useful to decompose a stochastic process in a finite interval on the real line as an infinite linear combination of orthogonal functions in $L^{2}[a, b]$. In this paper, a multi-dimensional non-linear stochastic differential equation driven by the Rayleigh process is the subject of investigations. Since the closed-form solution to the non-linear stochastic differential equations is not possible, the matrix-vector case arises, and decomposing the Rayleigh process in an infinite linear combination will introduce formidable complexity. Thus, the KL expansion to the estimation of non-linear dynamic systems is very scarce. 
Ravish Himmatlal Hirpara received his B.E. degree in Electrical Engineering from Saurashtra University, Rajkot, Gujarat, India in 2006 and M. Tech. degree in Electrical Engineering with specialization in Industrial Electronics from Sardar Vallabhbhai National Institute of Technology, Surat, Gujarat, India in 2008 and Ph.D. degree from Sardar Vallabhbhai National Institute of Technology, Surat, Gujarat, India in 2016. Currently, He is working as a Teaching Assistant in Electrical Engineering department of Sardar Vallabhbhai National Institute of Technology, Surat, Gujarat, India. His research includes stochastic differential systems and stochastic estimation and filtering of dynamical systems. He is IEEE Young Professionals, IEEE member, IEEE Control Systems Society, IEEE Circuits and Systems Society, IFAC Affiliate Member etc.

Shambhu N. Sharma received the BE degree from Government College of Engineering, Rewa (M.P.), India in 1994, the M Tech degree from Banaras Hindu University (Now IIT BHU), UP, India in 2000, and the PhD degree from Delhi University in 2007. Currently, he is working as Associate Professor in Electrical Engineering Department of the National Institute of Technology, Surat, India. He specializes in the areas of stochastic systems, control theory, stochastic filtering, and stochastic differential equations with applications to electrical and electronic networks. One of his works is known as a pioneering work in stochastic systems. A stochastic system bears his name, the Sharma-Parthasarathy stochastic two-body problems. He has published over 20 Journal papers in reputed Journals, which include International Journal of Control, Automatica, Royal Society Proceedings, Non-linear Dynamics, Journal of the Franklin Institute, AMC etc. He has also published about 12 papers in international conferences and symposia. He is the Author and co-Author of 4 solicited book chapters. He is a member of IEEE, ASME, ISCIE, IETE, IET, ACDOS, etc. 